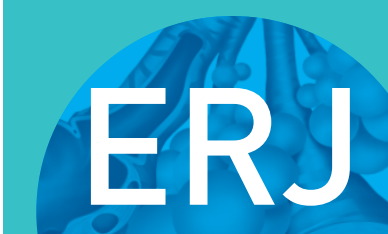

open research
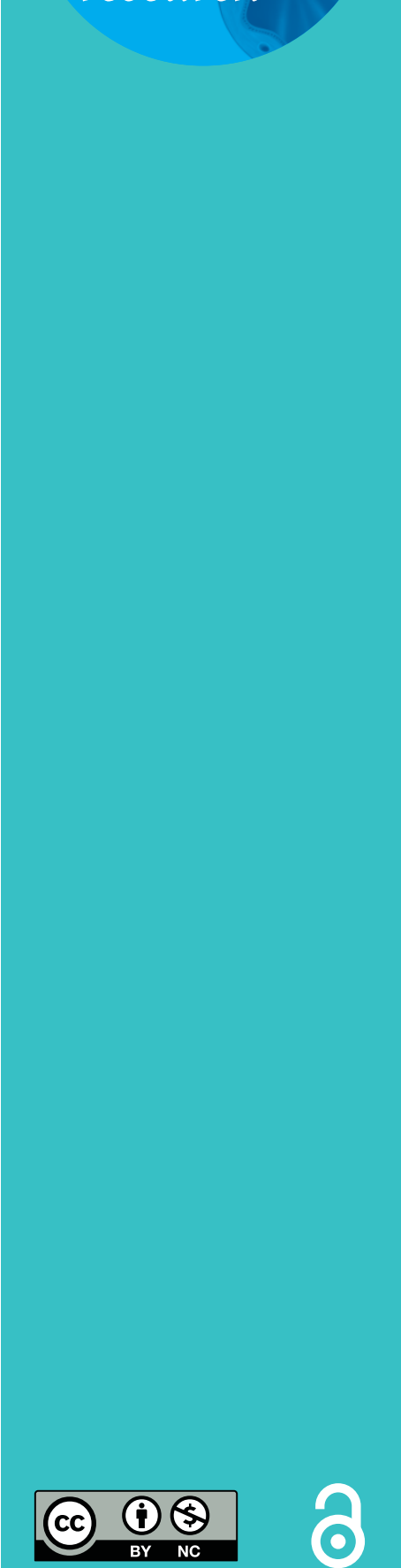

\section{Improving spirometry testing by understanding patient preferences}

\author{
Barbara Johnson ${ }^{1}$, Irene Steenbruggen $\mathbb{1}^{2}$, Brian L. Graham ${ }^{3}$ and \\ Courtney Coleman ${ }^{1}$
}

Affiliations: ${ }^{1}$ European Lung Foundation, Sheffield, UK. ${ }^{2}$ Lung Function Dept, Isala Hospital, Zwolle, The Netherlands. ${ }^{3}$ Respiratory Research Centre, Division of Respirology, Critical Care and Sleep Medicine, University of Saskatchewan, Saskatoon, SK, Canada.

Correspondence: Courtney Coleman, European Lung Foundation, 442 Glossop Road, Sheffield, S10 2PX, UK. E-mail: courtney.colemandeuropeanlung.org

ABSTRACT The American Thoracic Society and European Respiratory Society commissioned a task force to update the technical standards for spirometry testing with the aim of increasing the accuracy, precision and quality of spirometry measurements and improving the patient experience.

To inform the task force with patient experiences, the European Lung Foundation, in collaboration with the task force, conducted an online survey in 10 languages between August and September 2018.

There were 1760 respondents from 52 countries. The majority were adults (97.1\%); the most common reasons for spirometry referral were diagnosis (35.0\%) and management of an ongoing condition (60.1\%). $53.2 \%$ reported regularly using inhalers. Respondents were very experienced with spirometry: $89.9 \%$ completed more than one test; $48 \%$ completed 10 or more tests. However, most reported not knowing what forced expiratory volume in $1 \mathrm{~s}\left(\mathrm{FEV}_{1}\right)$ means $(59.4 \%)$ and only $39.6 \%$ knew their most recent $\mathrm{FEV}_{1}$; the exception was respondents with cystic fibrosis who reported much greater knowledge. Respondents rated as moderately or seriously problematic: being told to keep blowing when they felt nothing is coming out (31.4\%), coughing (30.4\%), tiredness (26.3\%) and concern about shortness of breath (20.1\%).

Overall, respondents found spirometry to be acceptable; however, an important minority (17\%) found it difficult. Patients want clear information before, during and after the test, including information on stopping medications. Operators have an important role in increasing the ease of patients and changes to the testing environment can increase patient comfort. Patients want access to their results and want to understand how they relate to their individual health.

@ERSpublications

"Why do I need spirometry and what do my results mean?": a survey of patient experiences highlights the need for clear information before, during and after spirometry testing, and underlines the important role of operators https://bit.ly/35L1Huk

Cite this article as: Johnson B, Steenbruggen I, Graham BL, et al. Improving spirometry testing by understanding patient preferences. ERJ Open Res 2021; 7: 00712-2020 [https://doi.org/10.1183/ 23120541.00712-2020].

This article has supplementary material available from openres.ersjournals.com.

Received: 30 Sept 2020 | Accepted: 13 Nov 2020

Copyright $\odot$ ERS 2021. This article is open access and distributed under the terms of the Creative Commons Attribution Non-Commercial Licence 4.0. 


\section{Introduction}

Spirometry is the most common test of lung function [1]. Unlike most other laboratory tests, the patient's full cooperation, including maximal breathing efforts, is essential. Despite the ubiquity of this test, little is known about patients' experiences of completing a spirometry test. As part of an update of the technical standards for spirometry by the American Thoracic Society (ATS) and European Respiratory Society (ERS) [2], a survey of people who had experience of spirometry was undertaken. This survey sought to provide the task force developing the technical standards for spirometry with more objective data on patients' experiences and perceptions of spirometry in order to better address the needs of the patients. Patient feedback was incorporated into the technical standards [2] and the key messages of the patient experience survey that were included in the 2019 standards are available in appendix A1 of the supplementary material. The current article seeks to give more detail about patients' experiences of spirometry and their views about areas for improvement.

In this article, the term "respondent" is used for a person who answered the survey; "patient" is used for the person being tested, recognising that not all of them are patients; "operator" is the person conducting the test.

\section{Methods}

Members of the spirometry technical standards task force, patient representatives and the European Lung Foundation (ELF) designed an online questionnaire to understand patients' experiences and preferences for spirometry testing. This survey was carried out to assist and inform the task force in developing an update of the ATS and ERS technical standards for spirometry.

The online questionnaire was hosted on SurveyMonkey (www.surveymonkey.com) for 2 months (between August and September 2018). A snowball sampling method was used to disseminate the survey with the aim of reaching as many patients from as many countries as possible.

The existing ELF, ATS and ERS patient and organisational networks were primarily used to promote the survey using social media and targeted newsletters. In addition, professionals involved in the task force used their networks and contacts with local patient organisations and national professional societies to disseminate the survey to their members. The survey was subsequently shared with a range of organisations, including the ATS Patient Advisory Roundtable, Cochrane Airways, Canadian Lung Association, European Cystic Fibrosis Registry, Dutch Association of Lung Function Analysts (Nederlandse Vereniging van Longfunctieanalisten) and TB Alert. The challenge to this approach is that although specific groups of patients can be targeted in this way, there is no way of calculating the actual reach or response rate.

The survey was open to adults and children who have had spirometry testing, including people living with lung conditions and those that have had the test as part of screening, e.g. during work-related health checks. The survey was available in 10 languages: Dutch, English, French, German, Greek, Italian, Polish, Portuguese, Russian and Spanish. A copy of the survey is provided as appendix A2 of the supplementary material.

The survey had seven sections: 1) respondent demographics; 2) reason for referral to spirometry and current medication; 3) forced expiratory volume in $1 \mathrm{~s}\left(\mathrm{FEV}_{1}\right)$ (understanding and knowledge of their result); 4) experience of spirometry tests (frequency, location, and information and advice received); 5) spirometry preferences (mouthpieces, nose clips and test duration); 6) barriers and benefits of spirometry; and 7) suggestions for improvement.

Respondents were also asked to give more information about these topics in free-text boxes. The survey finished by asking respondents if they would like to share any additional comments or experiences of spirometry. At the end of the survey respondents were invited to sign up to receive the results of the survey via a separate form.

\section{Results}

The survey received 1760 responses. Questionnaire data was analysed using SPSS version 25 (IBM, Armonk, NY, USA). 43\% completed the questionnaire in English (supplementary table S1). English language free-text responses were analysed using thematic analysis and themes were validated for other language responses by team members. Questionnaire percentages are presented along with further details from the thematic analysis for each survey section.

\section{Respondent demographics}

While the majority of the responses were from Europe, patients from 52 countries around the world completed the questionnaire (supplementary table S2). The most frequent countries of residence were 
Germany (16.5\%), UK (16.1\%), Belgium (11.9\%), USA (10.9\%), Spain (10.9\%), Italy (10.1\%) and the Netherlands (8.5\%). Although the study was open to both children and adults, the majority of respondents were adults aged $\geqslant 18$ years $(97.0 \%)$ (supplementary table S3). 53.2\% reported regularly using preventer, rescue or reliever inhalers.

The reason for initial referral for spirometry was diagnosis (35.0\%) or an ongoing condition. Table 1 shows the reasons for attendance in detail; respondents could select more than one option.

The majority of respondents had their spirometry test in a hospital (66.7\%) or primary care $(13.4 \%)$ setting, with only $1.5 \%$ reporting home spirometry.

Experience of spirometry testing and understanding of FEV 1

Respondents were very experienced with spirometry testing; $89.9 \%$ had completed more than one spirometry test and $48 \%$ reported having had 10 or more spirometry tests. However, most reported not knowing what $\mathrm{FEV}_{1}$ means (59.4\%) and only 39.6\% said they knew their most recent $\mathrm{FEV}_{1}$. Patients with cystic fibrosis (CF) were more likely (73.5\%) than other conditions (e.g. asthma $44.9 \%$ and chronic obstructive pulmonary disease (COPD) 43.9\%) to understand $\mathrm{FEV}_{1}$ and have knowledge of their current $\mathrm{FEV}_{1}$ (75.2\% compared with $34.8 \%$ for asthma and $44.9 \%$ for COPD). Patients with CF complete spirometry more regularly as part of their ongoing management compared with other lung conditions and children with CF need to be tested more frequently to take account of their growth. Age may also play a part, as respondents with CF were more likely to be younger, and older adults tend to have lower levels of "health literacy" or the ability to obtain, process and understand basic health information [3]. However, it did not apply to other conditions: for all respondents, $63.4 \%$ of those aged $>50$ years reported knowing what $\mathrm{FEV}_{1}$ meant compared with $53.22 \%$ of those aged $\leqslant 50$ years.

Patients want to be able to understand their results and what they mean for them. Many reported not understanding how their spirometry results relate to their specific condition. They would like to be able to have access to these results (either on paper or available digitally) and be able to compare them with their previous results. Patients would also like to understand how their results rate for someone with their lung condition (56.3\%), and how they rate for a healthy person of their age and height (53\%).

While the majority reported that the difficulty of performing spirometry was acceptable to them, a minority (17\%) did find it difficult (13.6\% responding "somewhat acceptable" and $3.4 \%$ responding "not at all acceptable"), and this was especially so for respondents with idiopathic pulmonary fibrosis $(26.7 \%$; $22.5 \%$ responding "somewhat acceptable" and $4.2 \%$ responding "not at all acceptable"). Some were concerned about the test $(20.1 \%$ rated as a moderate or serious problem to them "Concern about whether the test would cause shortness of breath"; $17.5 \%$ rated as moderate or serious "Concern about whether I could do the test") and in the qualitative data some expressed feeling anxiety about the results. Some felt vulnerable and found the process embarrassing (9.7\% rated as a moderate or serious problem to them "Feeling embarrassed performing the test"), especially when they found the test hard to do or when they produced sputum or excess saliva, as reported in the free-text comments.

Most tests lasted between 5 and $15 \mathrm{~min}$ (51.5\%), including the time for meeting, instructions and performing the test, although $32.1 \%$ lasted $>15 \mathrm{~min} .88 .8 \%$ of respondents found the length of the test acceptable.

\section{TABLE 1 Reasons for initial spirometry referral}

Investigating a problem with your breathing/to get a diagnosis

Chronic obstructive pulmonary disease

Asthma

Cystic fibrosis

Pulmonary fibrosis (for interstitial lung disease)

Bronchiectasis

Idiopathic pulmonary fibrosis

Occupational health testing

Pre-operative testing as part of your work-up for surgery

Lung cancer

$18(1.0)$

Neuromuscular disease

$13(0.7)$

Data are presented as $n(\%)$. Respondents could select more than one option (total responses $n=1786$ ). 
$14.1 \%$ of participants reported not getting information about spirometry at the time of testing and $38.3 \%$ reported that they did not receive any information about withholding inhalers prior to the test.

\section{Spirometry preferences}

The majority of patients would prefer to receive information about spirometry verbally (59.4\%), but many also wanted a printed handout $(40.6 \%)$. A smaller but significant proportion preferred to receive information electronically (28.1\%), especially those aged $18-50$ years. Patients would like to know what to expect from the test and what the potential benefits of completing the test are. $65.2 \%$ would like to understand what it means if their result has changed from the last time they did spirometry.

Many had used only one type of mouthpiece (43.4\%) or had no preference about the mouthpiece (18.4\%). The majority had used a nose clip (85.3\%), with $16.6 \%$ reporting nose clip comfort as a moderate or serious problem for them (10.6\% and $6.0 \%$, respectively). Many of the patients who reported anxiety in the free-text comment sections about their experience of spirometry cited the nose clip as problematic.

\section{Benefits and barriers of spirometry}

When asked about perceived benefits of spirometry, the most common rated benefits were improved knowledge of lung function (62.5\%) and getting a diagnosis (42.4\%).

Participants were asked to rate how problematic potential issues with spirometry were for them on a four-point Likert scale (not, minor, moderately and severely problematic) with a "not applicable" option. The issues that were rated most frequently as moderately or severely problematic are presented in table 2 . Many of these issues are reflected in the qualitative narrative in the following section.

\section{Overall view about spirometry and ideas for improvement}

Respondents were asked to provide feedback about their views on spirometry and any suggestions for improvement. 1422 respondents completed this section of the survey with responses varying from a few words to very long responses. The answers in English were coded for themes and validated in other languages; the themes are described in the following subsections.

\section{A necessary discomfort}

Although many respondents gave suggestions about how spirometry testing could be improved, it is important to reflect that many people find spirometry testing acceptable and not problematic: "It's a quick painless test. What's to improve?". While some respondents found the test uncomfortable, they felt it was a necessary and temporary discomfort: "I know some people don't like it ... but I see it as a necessity". For a small proportion of respondents, spirometry is very worrisome or "causes extreme anxiety". The operator should be mindful of this and have strategies for those who find the test stressful or anxiety inducing (such as practising before the test): "I would like for health professional[s] to listen to my concerns and at least show concern".

\section{TABLE 2 Respondents rating potential issues about spirometry testing as moderately or} severely problematic

To keep blowing even though you do not feel anything is coming out

Data are presented as \%. 
The importance of the operator

Many respondents emphasised the importance of the operator. Those who have had many tests feel it makes a real difference how friendly and encouraging the operator is: "a sympathetic, helpful and considerate nurse can do wonders during this test". Some felt the operator needed to fulfil the role of a cheerleader and that it made a difference to their results: "even if I have done it so often, I really need the health assistants' instructions and encouragement". Patients also felt operators need to "have empathy before, during and after the test", and that it is important to check if the patient is ready for the next blow and how they feel about having the test.

Patients also felt that it was important that operators did not express frustration when patients struggle to perform spirometry: "it would help if the technician/therapist did not express disappointment when I have trouble completing the test". Although many felt encouragement or coaching is important, some felt that "perhaps a gentler approach" than shouting instructions to blow was needed.

Clear guidance about inhaler use and performing the test

$38.3 \%$ of respondents did not receive advice about withholding inhalers prior to the test and this was reflected in the qualitative data, with many patients reporting they were not given clear guidance about using their inhaler before the test. It was suggested that they would like a reminder a week before the test of when to stop taking their inhaler in preparation for spirometry and which respiratory medications they should continue to use.

Respondents said it is really important to feel prepared for what is going to happen during the test and then be talked through the process. This knowledge may be taken for granted, particularly if the patient does regular spirometry tests, but it should not be. The process should be explained before each test: "my results are best when each test is explained at the start, and each step is called out to me during the test".

\section{Changes to the testing environment and process}

Respondents suggest that some small changes can make the process much more pleasant, e.g. providing "sputum pots, water and tissues without the need to ask". Patients would like a recovery period between tests and not to feel rushed, and have somewhere to sit and recover for a few minutes afterwards: "not a good idea to walk distances or drive when feeling light-headed or dizzy afterwards". Some patients would like somewhere "more private" when completing the test as they have concerns about infection due to their lung condition or feel embarrassment due to difficulty in completing the test. Some respondents suggested that better adjustability of machines would be positive as sitting awkwardly is not conducive to achieving good results.

\section{Access to results and their meaning}

Patients want to understand why they need a spirometry test, what their results are and what they mean for them. They would like to have access to their results (either on paper or available digitally) "without asking" and be able to compare them with their previous results. Some respondents requested a comparison with norms for a healthy person with the same age/height/weight or for someone with their condition. Patients would like these results and explanations without having to ask for them, and prefer that operators not "presume I understand what the score means". Patients would also like to know if there is anything they could do to improve their results. They feel this would be important information at the end of a spirometry test and suggest that operators "talk about results with me rather than waiting for my consultant to do so". Patients can be better supported to access and interpret their results: "I always have to ask for my results and ... feel like I'm being a nuisance asking for them. I have no understanding of the context of my results i.e. how I compare to others of my age with my condition? Are my results viewed as good or bad?".

\section{Discussion}

This large, multicountry survey of patients' experience of spirometry suggests that, for the most part, people perceive spirometry as a necessary test that they tolerate well. Respondents suggested some areas of improvement and these were consistent across country of residence. These were: importance of clear information before, during and after the test; operator empathy; and changes to the testing process and environment.

\section{Clear information}

This study highlights some areas of best practice around providing information; while most people prefer a face-to-face explanation, it is important to have written information available. A significant proportion of respondents reported confusion about withholding their inhaler prior to the test. To reduce the risk of patients unnecessarily stopping essential treatment, clear information about which respiratory medications 
should be withheld and for how long should be provided with the time of appointment, and a reminder should be considered.

Patients want to know what their result means and, for those having repeat tests, access to their previous result would also be beneficial. However, in many facilities conducting spirometry, the policies and procedures do not permit the operator to divulge this information. Since operators are well positioned to discuss measurements of lung volumes with the patients, and since many operators are also trained in patient education, spirometry facilities should consider reviewing and revising their policies regarding the information that operators may discuss with patients without crossing the line into interpretation of the results or diagnosis.

In addition, the ELF factsheet, "Testing your lungs: spirometry" [4], was coproduced with patients and addresses many of the common questions raised by survey respondents, including how to prepare for the test, what the results mean and what happens if the results are normal or abnormal. The factsheet is available in 15 languages and professionals can download it for use in their clinics [4].

\section{Operator empathy and changes to test procedure}

Some patients suggested that over-coaching was off-putting and stressful, and a gentler approach by operators may be warranted while still being their "cheerleader". Respondents valued privacy, time between tests to recover and a place to sit afterwards, and provision of supplies such as tissues and a sputum pot.

Thought should be given to those who find the test stressful or anxiety provoking to ensure that they do not avoid their spirometry tests. Suggestions such as having a practice blow or time to get used to the nose clip may help those who are feeling overwhelmed.

Based on the survey responses, the authors have compiled a number of tips for healthcare providers when conducting spirometry tests (table 3 ).

\section{Study strengths}

This study was designed with patients and received a large number of responses across many countries in 10 languages. It provides a clear picture of patients' experience of spirometry testing and how this can be improved. By seeking to engage patients and gather views widely from across many countries, through surveys such as this, we are able to provide a valuable explorative approach and highlight areas for future research.

\section{Limitations}

There are several methodological challenges to gathering patient perspectives. Our approach to gathering patient responses was pragmatic, aiming to maximise the number of responses within the timeframe and resources available. Very few responses were received from Africa, Asia and South America.

Challenges include the self-selection bias of the approach used. Individuals who complete a survey tend to have had a very good or very bad experience and are keen to communicate this, which may result in fewer

\section{TABLE 3 Tips for healthcare providers when conducting spirometry tests}

Provide clear advice to patients in advance of the test, including the reasons for carrying the test out and whether they need to discontinue using medication and inhalers

Ensure that tests are carried out in a quiet, private space and prepare the room in advance, e.g. by providing sputum pots, tissues and water

Give clear verbal instructions to patients before and during the procedure, even if the patient is experienced in taking spirometry tests

Between manoeuvres in a testing session, always ask the patient if they are ready to proceed before continuing the series

Allow time for a practice test and avoid rushing patients who are anxious or having difficulty taking the test

Give encouragement to patients during the test but avoid shouting instructions

Allow patients sufficient time to recover after the test

Inform the patient of their lung volume values, if permitted by local protocols; if appropriate, provide a comparison with their previous results or predicted values

Healthcare professionals who interpret spirometry are encouraged to explain results to their patients and provide comparisons to normal values 
responses from the moderate middle ground and bias the findings [5]. In addition, computer literacy and internet access also limit those who are able to participate. By using patient organisations to help disseminate the survey, we may also have had a higher proportion of responses from well-informed patients who are highly engaged in their healthcare.

\section{Conclusions}

This study provides a wide-range of views about spirometry testing and suggestions for practice for both respiratory services and individual operators. While spirometry is well tolerated by most, small changes to the procedure may help those who find it stressful, and further information about results and the procedure would be welcomed by most.

Acknowledgements: The authors would like to thank the following European Lung Foundation patient representatives for their input into the design of the survey: Annette Brons (The Netherlands), Dimitris Kontopidis (Greece) and Jasmine Parkinson (UK). We would also like to thank the members of the European Lung Foundation patient organisation network, the American Thoracic Society Patient Advisory Roundtable, and the members of the European Respiratory Society and American Thoracic Society for their help in disseminating the survey.

Conflict of interest: B. Johnson was an employee of the European Lung Foundation during the conduct of the study. I. Steenbruggen has nothing to disclose. B.L. Graham has nothing to disclose. C. Coleman is an employee of the European Lung Foundation.

\section{References}

$1 \quad$ Ranu H, Wilde M, Madden B. Pulmonary function tests. Ulster Med J 2011; 80: 84-90.

2 Graham BL, Steenbruggen I, Miller MR, et al. Standardization of Spirometry 2019 Update. An Official American Thoracic Society and European Respiratory Society Technical Statement. Am J Respir Crit Care Med 2019; 200: $70-88$.

3 Chesser AK, Keene Woods N, Smothers K, et al. Health literacy and older adults: a systematic review. Gerontol Geriatr Med 2016; 2: 1-13.

4 European Lung Foundation. Testing your lungs: spirometry. 2018. www.europeanlung.org/assets/files/en/ publications/spirometry-en.pdf Date last accessed: September 16, 2020.

5 Ahmed F, Burt J, Roland M. Measuring patient experience: concepts and methods. Patient 2014; 7: 235-241. 